\title{
Revisiting Imaging and Pathology of Malignant Appearing Uterine Smooth Muscle Tumors: A Mistaken Identity of Leiomyosarcoma on MRI
}

\author{
Mark Juhl1, Martha-Gracia Knuttinen'2, Rajul Kothari ${ }^{3}$, John Groth ${ }^{4}$ \\ ${ }^{1}$ College of Medicine, University of Illinois, Chicago, USA \\ ${ }^{2}$ Department of Radiology, University of Illinois, Chicago, USA \\ ${ }^{3}$ Department of Obstetrics and Gynecology, University of Illinois, Chicago, USA \\ ${ }^{4}$ Department of Pathology, University of Illinois, Chicago, USA \\ Email: mark.e.juhl@gmail.com, mgk600@hotmail.com, rkotha1@uic.edu, jgroth1@uic.edu
}

Received 30 March 2014; revised 30 April 2014; accepted 7 May 2014

Copyright (C) 2014 by authors and Scientific Research Publishing Inc.

This work is licensed under the Creative Commons Attribution International License (CC BY).

http://creativecommons.org/licenses/by/4.0/

(c) (i) Open Access

\begin{abstract}
Uterine leiomyoma is a relatively common condition occurring in a majority of African American and Caucasian women by age 50. Magnetic resonance imaging of the pelvis with contrast is the modality of choice when evaluating uterine tumors. We report a case of a patient with a four-day history of intra-menstrual vaginal bleeding who underwent magnetic resonance imaging of the pelvis that suggested the tumor was a leiomyosarcoma, the malignant counterpart of leiomyoma. Therefore, the patient underwent pre-operative uterine artery embolization to decrease blood loss with exploratory laparotomy, resection of pelvic mass and dilation and curettage. Pathologic evaluation of the mass returned a diagnosis of leiomyoma. Magnetic resonance imaging evaluation of leiomyomata remains difficult. Further development of imaging parameters could improve diagnosis of benign uterine fibroids.
\end{abstract}

\section{Keywords}

Magnetic Resonance Imaging, Uterine Fibroid Embolization, Fibroid, Leiomyoma, Leiomyosarcoma

\section{Case Report}

A 37-year-old female, G3 P2012, presented for consultation at our interventional radiology clinic regarding her 
magnetic resonance imaging (MRI) results showing a large pelvic mass. Family history was significant for her mother having her "pelvic" organs removed secondary to abnormal bleeding. However, no documented history of ovarian or breast cancer was noted. The patient presented to the emergency room a few days earlier with complaints of marked vaginal bleeding for several days. The clinical examination at that time was positive for a moderate amount of clotted blood in the vaginal vault and a firm, non-tender, palpable, left lower quadrant and supra pubic mass. The patient was discharged with a plan for consultation with the interventional radiology department for consideration of possible uterine fibroid embolization (UFE) after undergoing an MRI of her pelvis.

The MRI showed a large exophytic mass arising from the left aspect of the uterine body. The mass measured $18 \times 13.5 \times 10 \mathrm{~cm}$. The T2 weighted images of the mass showed heterogeneous mixed signal intensity with areas of hyperintensity (Figure 1(a), Figure 1(b)). On the post contrast T1 weighted-images, a central area of moderate enhancement following contrast injection surrounded by a rim of poorly enhancing tissue was noted (Figure 2). Sarcoma was the diagnosis of exclusion given the size and signal heterogeneity of the mass.

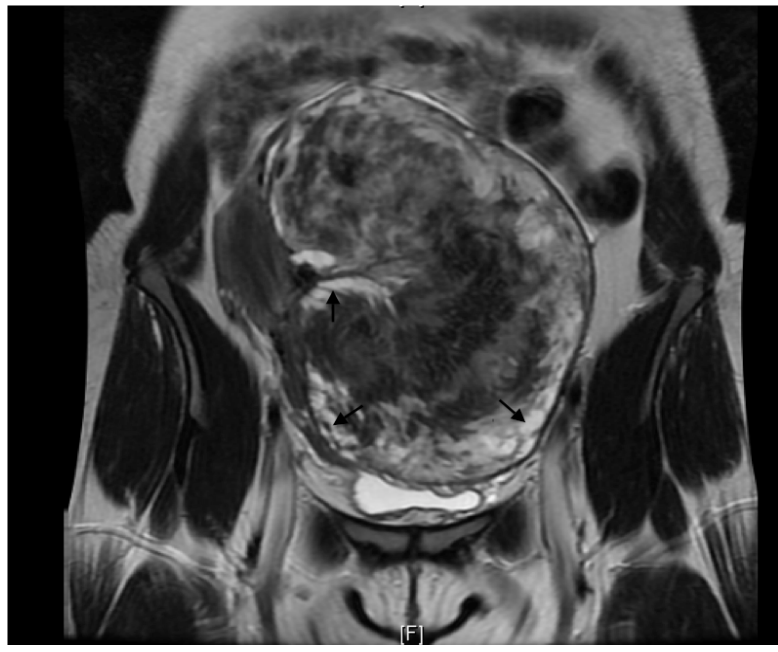

(a)

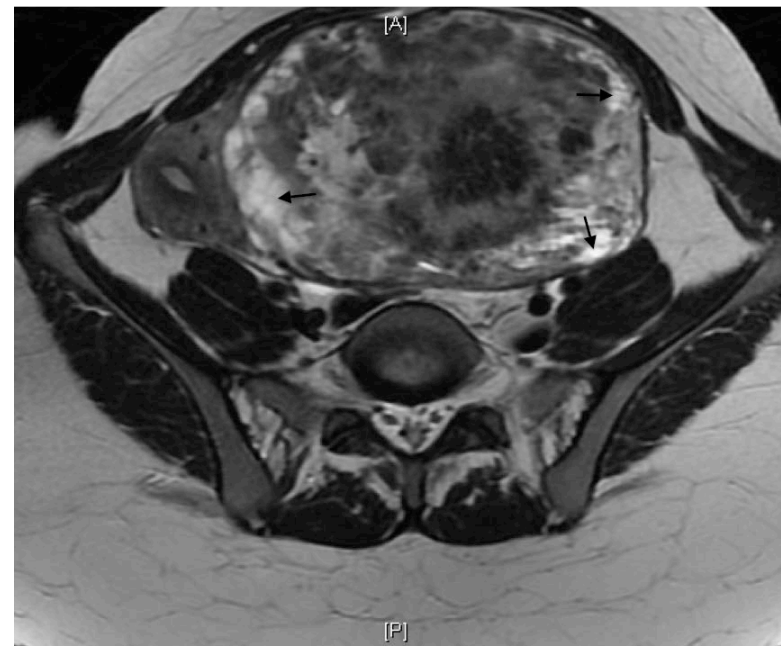

(b)

Figure 1. 37-year-old female with leiomyoma. Non-contrast coronal (a) and axial (b) T2 weighted MRI of the pelvis showing a mass with mixed signal intensity with areas of hyperintensity (Arrows).

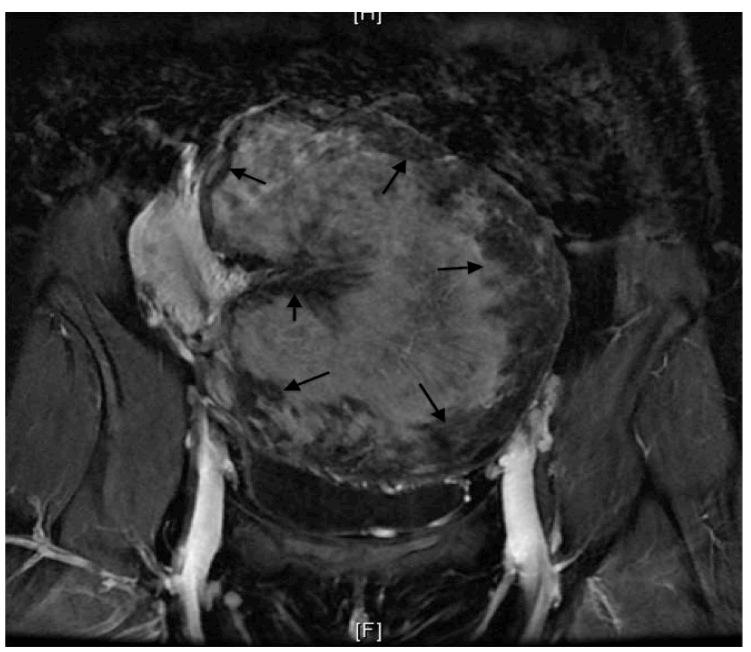

Figure 2. 37-year-old female with leiomyoma. Post contrast coronal T1 weighted MRI of the pelvis showing central enhancement of the mass with a rim of poorly enhancing tissue (Arrows). 
In response to MRI findings, it was decided not to immediately proceed with UFE and instead consult the gynecologic oncologist due to the concern for leiomyosarcoma. The opinions of the interdisciplinary team concluded that the best option for this patient was that she undergo surgical exploration with possible hysterectomy and staging if the tissue showed malignancy.

The patient underwent exploratory laparotomy, removal of pelvic mass, and dilation and curettage. Upon surgical exploration, a large left broad ligament mass was seen, adherent to both fallopian tubes and the anterior aspect of the uterus. The large mass deviated the fallopian tubes and uterus towards the right pelvis. The specimen was removed in its entirety and sent to pathology for intraoperative histopathologic evaluation using frozen section. The 1171 gram, specimen was lobulated, tan-pink, smooth and glistening with rubbery, tan-pink, whorled cut surfaces with scattered areas of hemorrhage and soft, yellow discoloration (Figure 3). Microscopic examination revealed elongated spindle cells, resembling myometrial cells, arranged in fascicles with eosinophilic cytoplasm and ovoid nuclei with intervening hyalinized stroma and thick walled vessels (Figure 4). Mitotic activity, coagulative necrosis, cellular atypia and myxoid change were absent. The patient then underwent a dilation and curettage with pathologic evaluation that was benign. The patient's post-operative course was uneventful and she was discharged on postoperative day two.

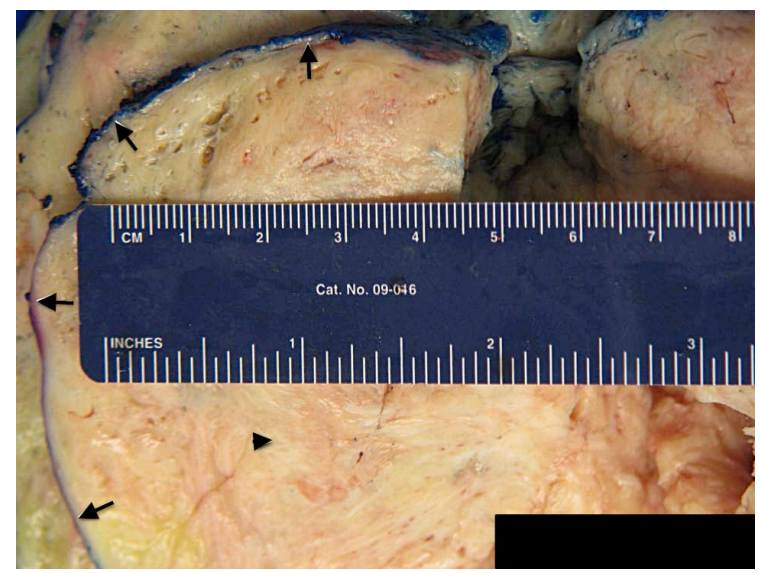

Figure 3. 37-year-old female with leiomyoma. Gross photography of the uterine mass demonstrating the well circumscribed nature of the lesion (Arrows) and whorled yellow-tan cut surface (Arrowhead).

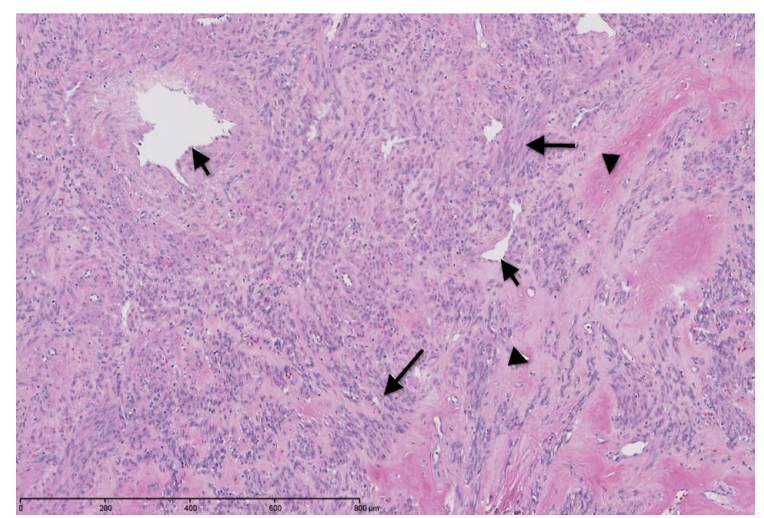

Figure 4. 37-year-old female with leiomyoma. Representative image of the uterine mass demonstrating the spindled cells with abundant eosinophilic cytoplasm, arranged in fascicles (Long arrows) and admixed with thick walled vessels (Short arrows) and areas of hyaliniza-tion (arrowheads). (Hematoxylin-eosin, original magnification $100 \times)$ 


\section{Discussion}

We report a case of a patient who underwent pre treatment MRI imaging to evaluate for possible UFE for the treatment of benign uterine leiomyoma. Her MRI imaging was quite concerning for a possible malignant appearing uterine sarcoma, and was therefore referred to gynecologic oncology. Given the massive size of her uterine tumor, she received pre-operative uterine artery embolization to prevent major blood loss, and underwent exploratory laparotomy, with resection of pelvic mass and dilation and curettage.

Uterine leiomyoma is a relatively common condition occurring in as high as $80 \%$ of African American women, and $70 \%$ of Caucasian woman by the age of 50 [1]. Smooth muscle lesions of the uterus are classified on a spectrum from typical leiomyoma to atypical leiomyoma to leiomyosarcoma, utilizing a compilation of pathologic findings including mitotic activity, differentiated cell type, necrosis, cellular atypia and invasion [2]. Furthermore, there is a vague category described as "smooth muscle tumor of uncertain malignant potential/STUMP", initially identified using mitotic activity alone, is now characterized as a smooth muscle tumor that cannot be reliably diagnosed as benign or malignant on the basis of current criteria [2] [3].

Treatment of leiomyosarcomas generally requires an aggressive, usually surgical approach with or without chemotherapy and/or radiation [4]. Uterine leiomyomas on the other hand, typically do not require treatment unless they become symptomatic.

The most common presenting symptom of patients with leiomyoma is abnormal uterine bleeding. Differentiating benign and malignant leiomyomata on MRI remains a challenging task. Typical MR characteristics of benign uterine leiomyomas include well-demarcated, homogenous, hypointense areas on T1 and T2-weighted images. Identification of a leiomyosarcoma remains difficult, although large heterogeneous masses with any areas of hyperintensity on T2 images, suggesting hemorrhage or coagulative necrosis, are thought to indicate sarcoma [5]-[7]. One small study found that the highest diagnostic accuracy in the diagnosis of malignant nonmyomatous uterine tumors occurred when three criteria were met: 1) a majority of the tumor had high signal intensity on T2 images; 2) areas of hyperintensity on T1 images; 3) and areas of non-enhancement following contrast administration [5]. These criteria were found to be $73 \%$ sensitive [5]. Of note is that our patient's MRI findings were in line with these criteria. In a different study, however, Cornfeld et al. found this set of criteria to not be as useful with a sensitivity of only 17\% [5] [8]. An additional objective criteria suggestive of a malignant tumor is the presence of irregular margins; the Cornfeld et al. study found this criteria to be $56 \%$ sensitive [8]. The MRI findings in our patient also documented this important criterion. Post contrast imaging of the masses generally have low signal intensity when benign, and high signal intensity when malignant [5] [6]. As seen from the literature, it is without question that strong objective criterion for accurately predicting uterine sarcomas has yet to be determined.

More recently, a study by Sato et al. investigated how the role of diffusion-weighted imaging (DWI) and apparent diffusion coefficient (ADC) can be used in differentiating leiomyoma from leiomyosarcoma [9]. They devised a diagnostic approach to the preoperative evaluation of leiomyomata in which patients could be stratified into high-risk and low-risk groups. If the masses exhibit low signal intensity on DWI, they were diagnosed as leiomyoma. If the signal intensity was intermediate or high, then ADC was measured. An ADC of 1.1 or greater would also then classify the mass as low-risk. An ADC $<1.1$ would place the patient in the high-risk group. Analysis showed a sensitivity of $100 \%$, with a specificity of $94 \%$ [9]. These numbers are promising, but further studies are needed to confirm these findings.

There are many conditions that may mimic leiomyosarcoma on imaging including endometrial carcinoma, lymphoma, intravenous leiomyomatosis, adenomyosis and myoma with secondary degeneration [6]. The pathologic diagnosis of leiomyosarcoma is based on cellular atypia, hypercellularity, high mitotic rate and the presence of necrosis. Cellular atypia and a high mitotic rate are unable to be discerned on MRI; however, increased cellularity and necrosis are noted to cause the high signal intensity on T2 weighted images. Another consideration would be to include lipoleiomyoma in the differential of this case. Lipoleiomyomas have an incidence of $0.03 \%-0.2 \%$, and are typically found in post-menopausal women [10]. Identification of fat within the uterine mass is the key to the diagnosis. The presence of fatty areas in these tumors leads to high signal intensity on T1 and low signal intensity on T2 weighted images. However, this was excluded as no high signal was noted on the T1 weighted imaging. Pathologic and histologic analysis also did not demonstrate any large component of adipose tissue.

The patient's imaging showed centrally located high signal intensity on T2-weighted images indicating possi- 
ble hypercellularity or necrosis. Additionally, post-contrast imaging of the mass revealed an enhancing center surrounded by a non-enhancing ring of tissue. These imaging parameters suggested the malignant counterpart; however the pathologic evaluation of the mass consisted of areas of hemorrhage, which most likely correlated with the hyperintensity noted on the T2 weighted images.

Imaging of a benign leiomyoma may mimic a leiomyosarcoma and should be considered in a differential diagnosis based on imaging. High clinical suspicion combined with suggestive imaging characteristics such as ill-defined margins and post-contrast high signal intensity, should prompt appropriate surgical consultation and possible intervention.

We conclude that MRI evaluation of uterine leiomyomata remains a necessary yet imperfect science. Further MRI parameters, such as the validation and further development of DWI and ADC, may shed light on better imaging diagnosis of benign appearing fibroids.

\section{References}

[1] Baird, D.D., Dunson, D.B., Hill, M.C., Cousins, D. and Schectman, J.M. (2003) High Cumulative Incidence of Uterine Leiomyoma in Black and White Women: Ultrasound Evidence. American Journal of Obstetrics \& Gynecology, 188, 100-107. http://dx.doi.org/10.1067/mob.2003.99

[2] Bell, S.W., Kempson, R.L. and Hendrickson, M.R. (1994) Problematic Uterine Smooth Muscle Neoplasms. A Clinicopathologic Study of 213 Cases. The American Journal of Surgical Pathology, 18, 535-558. http://dx.doi.org/10.1097/00000478-199406000-00001

[3] Tavassoli, F.A. and Devilee, P. (2003) World Helath Organization Classification of Tumours. Pathology and Genetics of Tumours of the Breast and Female Genital Organs. 3rd Edition, IARC Press, 233-242.

[4] Dinh, T.A., Oliva, E.A., Fuller Jr., A.F., Lee, H. and Goodman, A. (2004) The Treatment of Uterine Leiomyosarcoma. Results from a 10-Year Experience (1990-1999) at the Massachusetts General Hospital. Gynecologic Oncology, 92, 648-652. http://dx.doi.org/10.1016/j.ygyno.2003.10.044

[5] Tanaka, Y.O., Nishida, M., Tsunoda, H., Okamoto, Y. and Yoshikawa, H. (2004) Smooth Muscle Tumors of Uncertain Malignant Potential and Leiomyosarcomas of the Uterus: MR Findings. Journal of Magnetic Resonance Imaging, 20, 998-1007. http://dx.doi.org/10.1002/jmri.20207

[6] Rha, S.E., Byun, J.Y., Jung, S.E., Lee, S.L., Cho, S.M., Hwang, S.S., Lee, H.G., Namkoong, S.-E. and Lee, J.M. (2003) CT and MRI of Uterine Sarcomas and Their Mimickers. AJR, 181, 1369-1374. Http://dx.doi.org/10.2214/ajr.181.5.1811369

[7] Sahdev, A., Sohaib, S.A., Jacobs, I., Shepherd, J.H., Oram, D.H. and Reznek, R.H. (2001) MR Imaging of Uterine Sarcomas. AJR, 177, 1307-1311. http://dx.doi.org/10.2214/ajr.177.6.1771307

[8] Cornfeld, D., Israel, G., Marel, M., Weinreb, J., Schwartz, P. and McCarthy, S. (2010) MRI Appearance of Mesenchymal Tumor s of the Uterus. EJR, 74, 241-249. http://dx.doi.org/10.1016/j.ejrad.2009.03.005

[9] Sato, K., Yuasa, N., Fujita, M. and Fukushima, Y. (2014) Clinical Application of Diffusion-Weighted Imaging for Preoperative Differentiation between Uterine Leiomyoma and Leiomyosarcoma. American Journal of Obstetrics \& Gynecology, 210, 368.e1-8.

[10] Avritscher, R., Iyer, R.B., Ro, J. and Whitman, G. (2001) Lipoleiomyoma of the Uterus. AJR, 177, 856. http://dx.doi.org/10.2214/ajr.177.4.1770856 